\title{
Une nouvelle théorie globale de la lubrification des articulations
}

\author{
A new global theory on the lubrication of articulations
}

\author{
Richard Collins \\ Professeur associé à l'Université de Technologie de Compiègne \\ Département de Génie Biologique
}

\section{Introduction}

Les coefficients de frottement de l'ordre de 0,01 observés pour les articulations physiologiquement normales sont inférieurs d'un facteur de 10 à leurs homologues industriels. II parait essentiel de comprendre les mécanismes responsables du fonctionnement "idéal" de l'articulation naturelle afin de : (a) suggérer des thérapeutiques pour le traitement des ostéoarthroses qui semblent associées à des niveaux de frottement élevés, (b) améliorer les conceptions des prothèses qui devraient mieux se conformer aux mécanismes naturels.

Les résultats des travaux menés par des équipes de chirurgiens orthopédiques, d'ingénieurs mécaniciens et de biophysiciens, notamment en Angleterre et aux Etats-Unis depuis les années 1960 ont été décrits dans les œuvres de synthèse telles que celles de Wright (1969), Apley (1972), Freeman (1972) et Kenedi (1973).

Très schématiquement, on peut résumer le progrès atteint dans ce domaine comme il suit.

Mécanismes fondamentaux de fonctionnement des articulations humaines

Les études approfondies qui ont été poursuivies notamment en Angleterre, ainsi qu'aux Etats-Unis, semblent avoir écarté le mécanisme de lubrification purement hydrodynamique, au profit d'un régime mixte d'une couche lubrificatrice non-fluide de surface, avec la contribution d'un support par des "réservoirs" microscopiques de fluides empiégés dans la structure superficielle du cartilage articulaire.

Il reste à quantifier ces hypothèses en élaborant une théorie précise dont chaque élément trouve sa justification dans les observations d'une série d'expérimentations soigneusement effectuées au laboratoire.
Les hypothèses qui forment la base de l'analyse mathématique, présentée ici, portent sur le modèle suivant :

Lorsqu'une surface de l'articulation exerce une charge sur l'autre surface, l'espace entre les deux diminue progressivement jusqu'à quelques dizaines d'angstroms. La phase aqueuse du fluide synovial est exprimée, laissant une concentration locale de molécules de mucine dans la zone chargée. Au-delà d'un niveau critique de concentration, les molécules à longue chaîne ont alors tendance à s'ancrer sur les surfaces cartilagineuses, stabilisant ainsi la couche lubrificatrice "instantanée".

La zone de contact se déplace pendant la marche et la même procédure se reproduit à chaque nouvelle position, tandis que les molécules de mucine préalablement concentrées dans la zone déchargée par la suite sont ré-absorbées dans le fluide synovial. C'est ainsi que l'articulation se crée continuellement une "chaus" sée" lubrificatrice dans la direction de son déplacement.

A ce phénomène s'ajoute, pour des charges plus élevées, une exudation de fluide de la matrice du cartilage, lors de sa compression, modifiant ainsi l'épaisseur de la couche lubrificatrice.

En effet, le fluide synovial contenu dans les pores $\mathrm{du}$ cartilage subit deux forces opposées et développe, pour une compression du cartilage : a) une vitesse relative vers la surface due au déplacement de celle-ci vers l'os subchondral, et b) une vitesse vers l'os (en sens opposé au gradient de pression) conforme à la loi de Darcy pour un écoulement dans un milieu poreux. Lorsque l'articulation n'est plus soumise à la pesanteur, les vitesses changent de directions.

Dans le cadre de ce modèle il apparait que l'effet a) domine sur b), c'est-à-dire, le mouvement net des mucines est dirigé vers la surface du cartilage lorsqu'on appuie sur celui-ci. Ceci assure l'alimentation d'une couche lubrificatrice pendant la phase de charge et une réabsorption dans le cartilage lors de la décharge. 
Or l'effet b) dépend de la perméabilité du cartilage. Il est possible pour certains cas pathologiques caractérisés par une augmentation de la perméabilité (microfissuration ostéoarthrosique) que l'effet b) puisse dominer sur a), inversant ainsi les directions des flux à travers la surface du cartilage. Ces surfaces seraient alors privées de mucine lors de la mise en charge, et lubrifiées seulement lors de la décharge, ce qui est tout à fait contraire aux besoins.

Cette inversion peut marquer le début d'une dégénération mécanique de la surface cartilagineuse (fibrillation, ulcération) menant éventuellement à un état avancé de l'ostéoarthrose [voir aussi Freeman (1973)].

\section{Hypothèses sur la pathogenèse de l'ostéoarthrose}

La dégénération du cartilage pourrait être éventuellement déclenchée, en tout premier lieu, par des pertes du fluide contenu dans les pores de la matrice du cartilage. Un déséquilibre des transferts à travers la couche de cartilage rend celle-ci plus déformable, augmentant les contraintes dans les fibres de collagène, les brisant, et laissant partir ainsi les protéoglycans à travers les microfissures créées dans la surface cartilagineuse.

Il a été proposé par Freeman (1973) et Weightman (1976) que la première perte de fluide de la matrice cartilagineuse, qui est responsable de l'amorce de la fibrillation du cartilage, résulte de la "fatigue mécanique" des fibres de collagène. Ces fibres contiennent les protéoglycans qui eux contiennent du fluide. La charge articulaire est supportée à raison de $80 \%$ par ce fluide.

Weightman (1976) a donné les courbes expérimentales reliant les niveaux de contraintes intra-articulaires répétées qui sont suffisantes pour provoquer des microfissures après un certain nombre de cycles d'application. Mais le niveau réel des contraintes physiologiques est encore inconnu. Sa connaissance permettrait de conclure quantitativement sur le rôle de la fatigue comme mécanisme primaire de l'ostéoarthrose.

Récemment Mow et Mansour (1977) ont abordé une analyse unidimensionnelle des caractéristiques de relaxation du cartilage soumis à une charge et doté d'une perméabilité non-uniforme, en utilisant une méthode linéarisée sur l'hypothèse de déformations infinitésimales. Ces auteurs ont d'ailleurs infirmé eux-mêmes cette hypothèse en montrant par le calcul que la valeur obtenue pour les déformations n'autorise pas une telle linéarisation.

La theorie bi-dimensionnelle non stationnaire $\mathrm{du}$ modèle global décrit dans le paragraphe suivant lie la charge et la déformation du cartilage à l'analyse de la formation de gel lubrificateur dans l'espace intraarticulaire adjacent. On exploite directement les mesures récentes de Mansour et Mow (1976) effectuées en laboratoire et exprimant la dépendance entre la perméabilité du cartilage, la charge et la déformation en compression.

\section{Analyse mathématique}

Une analyse globale et relativement simple de la formation de couche lubrifiante de mucopolysaccharides sur les surfaces articulaires fournit une relation directe entre le cycle de charge, les propriétés physiques du cartilage et les composants du fluide synovial, et la concentration de molécules de mucine.

L'analyse se déroule en deux étapes: la première (Fig. 1) traite de l'échange de fluide (solvant) à travers les surfaces articulaires déformables, et la seconde (Fig. 4) traite des variations des concentrations de mucopolysaccharides qui en résultent dans l'espace articulaire adjacent lorsque les surfaces cartilagineuses se rapprochent. On émet l'hypothèse qu'une "couche" lubrificatrice de gel se formera momentanément lorsqu'un niveau critique de la concentration d'hyaluronate est atteint, et qu'il sera essentiel pour le bon fonctionnement de l'articulation que cette lubrification de surface soit renouvelée et maintenue correctement.

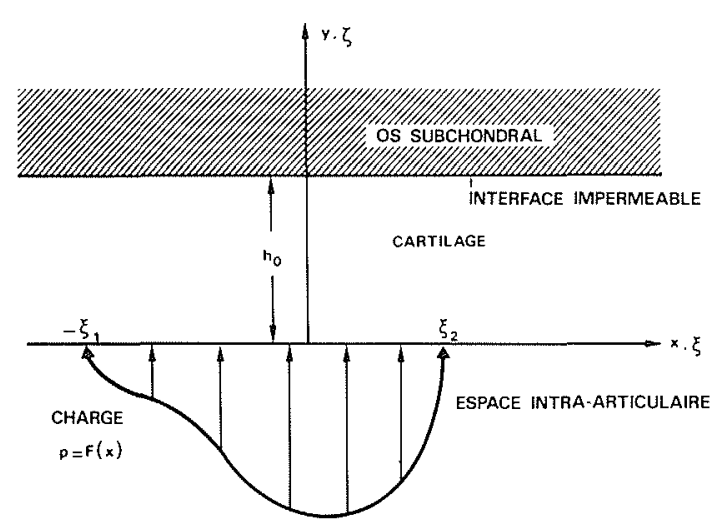

Figure 1 -Charge sur une surface de cartilage articulaire.

\section{Transport du fluide au sein du cartilage}

Les équations couplées du mouvement de la matrice déformable cartilagineuse et de la fraction du fluide mobile contenu dans ses pores peuvent s'écrire sous une forme légèrement modifiée par rapport à celles proposées par Nowinski (1971) et Torzilli et Mow (1976).

Matrice :

$$
\rho_{m} \frac{\partial^{2} \vec{u}^{\prime}}{\partial t^{\prime 2}}=\operatorname{div} \sigma_{m}-\left(\vec{u}_{t^{\prime}}^{\prime}-\vec{v}^{\prime}\right) / k^{\prime}(\vec{x})
$$

Fluide :

$$
\rho_{f} \frac{\overrightarrow{D v}^{\prime}}{D t^{\prime}}=\operatorname{div} \sigma_{f}-\left(\vec{u}_{t^{\prime}}^{\prime}-\vec{v}^{\prime}\right) / k^{\prime}(\vec{x})
$$

en fonction de la dérivée de Lagrange $\frac{D}{D t}=\frac{\partial}{\partial t^{\prime}}+\left(\vec{v}^{\prime} \cdot \nabla\right)$ où $\rho_{m}, \rho_{f}$ désignent respectivement les densités de la matrice et du fluide, et $k^{\prime}(\overrightarrow{\vec{x}})$ la perméabilité de la matrice 
cartilagineuse au fluide. Les tenseurs de contrainte de la matrice $\left(\sigma_{m}^{\prime}\right)$ et du fluide $\left(\sigma_{f}^{\prime}\right)$ sont donnés par les relations constitutives

$$
\begin{aligned}
& \sigma_{m}^{\prime}=p^{\prime} I+2 N^{\prime} \operatorname{def} \vec{u}^{\prime}+A \operatorname{div} \vec{u}^{\prime} \\
& \sigma_{f}^{\prime}=-p^{\prime} I+Q^{\prime} \operatorname{div} \vec{u}{ }^{\prime}
\end{aligned}
$$

en fonction des paramètres élastiques $N^{\prime}, Q^{\prime}, A^{\prime}$ (Biot et Willis 1957, Biot 1963) du cartilage et de la pression hydrostatique $p$. Le tenseur classique de déformation def $\vec{u}$ s'exprime sous la forme indiciaire suivante :

$$
\operatorname{def} \vec{u}^{\prime}=\frac{1}{2}\left[\operatorname{grad} \vec{u}^{\prime}+\left(\operatorname{grad} \vec{u}^{\prime}\right)^{*}\right]=\frac{1}{2}\left(\frac{\partial u_{k}^{\prime}}{\partial x_{l}^{\prime}}+\frac{\partial u_{l}^{\prime}}{\partial x_{k}^{\prime}}\right)
$$

L'équation (3.2) peut être considérée tout simplement comme la forme généralisée de la loi de Darcy (1856) décrivant l'écoulement non-stationnaire dans un milieu poreux déformable en fonction de la vitesse relative $\left(\vec{u}_{t}-\vec{v}\right)$ entre le cartilage comprimé et le fluide contenu dans ses pores.

Remplaçant (3.3) et (3.4) dans (3.1) et (3.2) et reformulant en fonction des nouvelles variables adimensionnelles (Fig. 1)

$$
\begin{array}{r}
\xi=\frac{x^{\prime}}{\bar{h}^{\prime}}, \quad \xi=\frac{y^{\prime}}{\vec{h}^{\prime}}, \quad \vec{u}=\vec{u}^{\prime} / \bar{h}^{\prime}, \quad k=k^{\prime} / \bar{k}^{\prime} \\
\vec{v}=\frac{\vec{v}^{\prime} h_{0}^{\prime}}{N \vec{k}^{\prime}}, \quad t=\frac{N^{\prime} k_{0}^{\prime}}{\vec{h}^{\prime 2}} t^{\prime}, \quad p=p^{\prime} / N^{\prime}
\end{array}
$$

$f=F / N^{\prime} \quad$ (fonction caracterisant la charge) du cartilage et sa perméabilité, on obtient la forme adimensionnelle des équations régissant les mouvements

Matrice :

$$
\begin{array}{r}
\frac{N^{\prime} \bar{k}^{\prime 2} k \rho^{\prime}}{\bar{h}^{\prime 2}} \frac{\partial^{2} \vec{u}}{\partial t^{2}}=k\left[\nabla p+\nabla^{2} \vec{u}+\frac{N^{\prime}+A^{\prime}}{N^{\prime}} \nabla(\nabla \cdot \vec{u})\right] \\
-\left(\vec{u}_{t}-\vec{v}\right)(3.5)
\end{array}
$$

Fluide :

$\frac{N^{\prime} \bar{k}^{\prime 2} k \rho_{f}^{\prime}}{\bar{h}^{\prime 2}} \frac{\overrightarrow{D v}}{D t}=k\left[-\nabla p+\frac{Q^{\prime}}{N^{\prime}} \nabla(\nabla \cdot \vec{u})\right]+\left(\vec{u}_{t}-\vec{v}\right)$

Les densités du fluide et du cartilage sont toutes deux approximativement égales à celle de l'eau. Pour le cartilage normal placé dans une solution de Ringer, Hori et Mockros (1976) déterminent la valeur du module de cisaillement $G=2.67 \times 10^{7} \mathrm{dyn} / \mathrm{cm}^{2}$, et le coefficient de Poisson $\nu=0,473$. On peut déduire une valeur moyenne de la perméabilité $\bar{k}=10^{-12} \mathrm{~cm}^{4} / \mathrm{dyn}-\mathrm{sec}$ d'après les mesures récentes de Mansour et Mow (1976), qui paraît relativement supérieure à celle de Mc Cutchen (1962) pour la même pression. Enfin, on évalue pour une couche typique de cartilage d'épaisseur $0,2 \mathrm{~cm}$ le terme adimensionnel $N^{*} \bar{k}^{\prime 2} / \bar{h}^{2}$ apparaissant dans les membres gauches des équations (3.5) et (3.6). Il est de l'ordre de $10^{-7}$, et donc de plusieurs ordres de grandeur en-dessous du membre droit. Par conséquent, on élimine les dérivées par rapport au temps. Il en résulte que le comportement du cartilage soumis à une charge variable dans le temps, peut être décrit par une séquence d'états quasi-statiques, où le temps figure seulement comme paramètre. En d'autres termes, pendant la marche normale, la réponse du cartilage est pratiquement instantanée vis-à-vis de l'évolution temporelle du niveau de la charge qui est beaucoup plus lente.

On doit souligner ici que les équations qui en résultent :

Matrice :

$$
k\left[\nabla p+\nabla^{2} \vec{u}+\mathscr{T} \nabla(\nabla \cdot \vec{u})\right]-\left(\vec{u}_{t}-\vec{v}\right)=0(3.7)
$$

Fluide :

où :

$$
\begin{gathered}
k[-\nabla p+\mathscr{2} \nabla(\nabla \cdot \vec{u})]+\left(\vec{u}_{t}-\vec{v}\right)=0 \\
\mathscr{\alpha}=\frac{N^{\prime}+A^{\prime}}{N^{\prime}}, \mathscr{\mathcal { O }}=Q^{\prime} / N^{\prime}
\end{gathered}
$$

représentent seulement la déformation d'un système diphasique fluide-cartilage. Des mesures de la perméabilité et des coefficients de diffusion de différents composants du fluide synovial contenu dans le cartilage, Maroudas (1973) a démontré que les grosses molécules de protéoglycans, qui sont essentielles pour assurer la lubrification des articulations, ne peuvent se déplacer librement à travers la matrice cartilagineuse. Par conséquent, on les prendra en compte uniquement dans la seconde phase de l'analyse qui traitera de leur transport dans l'espace intra-articulaire.

Les conditions aux limites s'expriment sous forme adimensionnelle :

a) à la surface du cartilage, prise nominalement à $\zeta=0$ (Fig. 1)

$$
\begin{aligned}
p & =f(x, t) & & \text { pour } \quad \xi_{1}<\xi<\xi_{2} \\
& =0 & & \text { au-delà de l'espace chargé }
\end{aligned}
$$

$$
|\xi|>\left|\xi_{1}\right|,\left|\xi_{2}\right|
$$

b) à la jonction du cartilage avec l'os subchondral $\zeta=1$, qui est largement admise comme rigide et imperméable au fluide (pour le cartilage adulte), on déduit de (3.8) que :

$$
\frac{\partial p}{\partial \zeta}=0 \quad \text { pour } \quad|\xi|<\infty
$$

On peut alors introduire la notion de dilatation $e$ du cartilage :

$$
e=\operatorname{div} \vec{u}
$$

et supposer en première approximation, que les variations locales de la perméabilité sont relativement faibles (Maroudas 1967), ainsi que les constantes d'élasticité $\mathcal{O}$ et $\mathscr{Q}$ à l'intérieur de la couche cartilagineuse.

En sommant les équations (3.7) et (3.8), on élimine la pression ainsi que la vitesse du fluide, et en calculant la divergence, on obtient l'équation de Laplace pour la dilatation (Torzilli et Mow 1976).

$$
\nabla^{2} e=0
$$

Bien que la relation (3.11) ne soit rigoureusement variable que pour une perméabilité uniforme du cartilage, on peut cependant aboutir à une estimation du champ des pressions, avec une bonne précision qui rend compte des faibles variations de la perméabilité associées à des variations importantes de la dilatation, ceci avec une marge d'erreur de l'ordre de $d / d y(\log k)$. 
4 cet effet, on peut établir une relation empirique sin sle entre la variation de la perméabilité du cartilage et la pression hydrostatique d'après les résultats des expériences récentes de Mansour et Mow (1976). Ces auteurs ont fait varier de façon indépendante le gradient de pression et la déformation en compression durant les mesures de la perméabilité de cartilage articulaire bovin grâce à un appareil spécialement conçu. Comme on pouvait le présager, la perméabilité décroit lors d'une augmentation de la pression imposée, suite à une compression des pores du cartilage. (cf. Fig. 2 qui a été construite en moyennant leurs divers résultats).

On a trouvé que la perméabilité du cartilage varie linéairement en fonction de la dilatation.

$$
k=k_{0}-k_{1} e
$$

alors que la variation de cette perméabilité en fonction de la pression imposée peut être décrite par une hyperbole ayant des asymptotes parallèles aux axes des coordonnées

$$
(k-\widetilde{k}) p=c \text { ste }=c
$$

ou $\mathcal{k}=0,25, c=0,12$ et $k_{0}, k$ varient en fonction de la pression imposée (Fig. 3). En éliminant $k$ dans les équations (3.12) et (3.13), on aboutit à :

$$
\begin{gathered}
e=\alpha-\frac{\beta}{p} \\
\text { où : } \quad \alpha=\frac{k_{0}-\widetilde{k}}{k_{1}} \text { et } \beta=c / k_{1}
\end{gathered}
$$

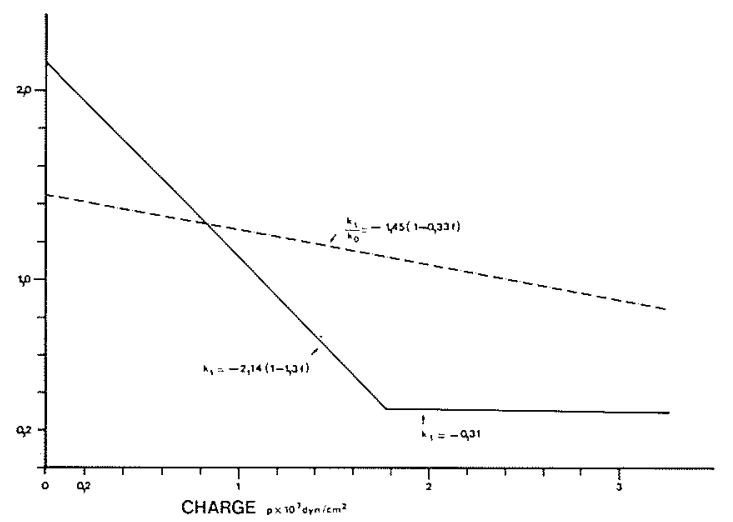

Figure 2 - Variations des coefficients de la perméabilité en fonction de la charge.

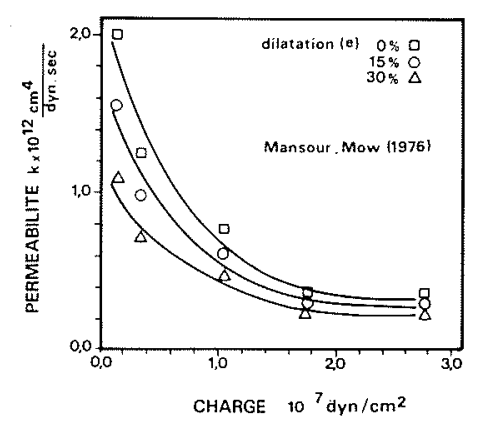

Figure 3 - Variations mesurées de la perméabilité en fonction de la dilatation (déformation).
II suffit maintenant de remplacer cette relation empirique (3.14) dans l'équation fluide-matrice (3.11) afin d'obtenir une seule équation différentielle linéaire de deuxième ordre en fonction de la pression sous la forme :

$$
\nabla^{2}\left(\frac{1}{p}\right)=0
$$

qu'il faut résoudre en tenant compte des conditions aux limites mixtes (3.10).

On peut remplacer :

$$
\mathscr{P}_{0}=1 / p
$$

dans (3.15) et (3.10).

Le problème équivalent de von Neumann peut être résolu de manière directe en utilisant une transformation intégrale de Fourier par rapport à $\xi$

$$
\widetilde{\mathscr{P}}(\omega, \zeta) \equiv \frac{1}{(2 \pi)^{1 / 2}} \int_{-\infty}^{\infty} e^{-i \omega \xi} \mathscr{P}_{\Lambda}(\xi, \zeta) d \zeta(3
$$

pour que (3.15) devienne tout simplement, en fonction de la variable transformée $\mathscr{P}$.

$$
\frac{d^{2} \widetilde{\mathscr{T}}}{d \zeta^{2}}-\omega^{2} \widetilde{\mathscr{P}}=0
$$

avec :

$$
\widetilde{\mathscr{P}}(0)=1 / \widetilde{f}, \quad d \widetilde{\mathscr{P}} / d \zeta
$$$$
(1)=0
$$

d'où : $\quad \widetilde{\mathscr{T}}=\frac{1}{\widetilde{f}(\omega)} \operatorname{ch}(1-\zeta) \omega / \operatorname{ch} \omega$

Après inversion, on obtient la répartition des pressions dans le plan physique sous la forme :

$$
\mathscr{P}_{2}(\xi, \zeta)=\sin \frac{\pi}{2} \zeta \quad \int_{-\infty}^{+\infty} \frac{1}{f(\zeta)} \frac{\operatorname{ch} \pi / 2\left(\xi-\xi^{\prime}\right) d \xi^{\prime}}{\operatorname{ch} \pi\left(\xi-\xi^{\prime}\right)-\cos \pi \zeta}
$$

en fonction de la charge $f(\xi)$ dans la zone de contact entre les surfaces articulaires opposées.

Les mesures expérimentales de cette fonction sont actuellement en cours, mais en l'absence d'informations plus précises, on considère une répartition de la charge dans la zone de contact $-\xi_{1}(t)<\xi<\xi_{2}(t)$, c'est-à-dire

$$
f(\xi, t)=F(t)
$$

où l'amplitude $F$ de la charge ainsi que l'importance $\left|\xi_{1}\right|+\left|\xi_{2}\right|$ de la zone de contact, sont des fonctions paramétriques du temps.

Appliquant (3.21), l'intégration du membre droit de (3.20) donne :

$$
\begin{aligned}
& \mathscr{P}_{R}(\xi, \zeta ; t)=\frac{1}{p(\xi, \zeta ; t)}=\frac{1}{\pi F(t)} \times \\
& \left.\operatorname{arctg} \frac{\operatorname{sh} \frac{\pi}{2}\left(\xi+\xi_{1}\right)}{\sin \frac{\pi}{2} \zeta} \operatorname{arctg} \frac{\operatorname{sh} \frac{\pi}{2}\left(\xi-\xi_{2}\right)}{\sin \frac{\pi}{2} \zeta}\right]
\end{aligned}
$$

Remplaçant cette solution pour la pression $p$ dans l'équation (3.8) du mouvement du fluide conduit à une expression de la vitesse relative

$$
\left(\vec{v}-\vec{u}_{t}\right)=-k(\nabla p-2 \nabla e)
$$


Après avoir éliminé $e$ en utilisant (3.14), on obtient

$$
\left(\vec{v}-\vec{u}_{t}\right)=-k \nabla p \quad\left(1-\mathscr{S} \frac{\beta}{p^{2}}\right)
$$

C'est particulièrement la composante normale de la vitesse relative qui détermine le flux de fluide entrant du cartilage dans l'espace intra-articulaire. A partir du déplacement instantané de la surface $u=u$ et des conditions aux limites (3.4), on peut évaluer la composante normale du gradient de pression $\nabla p$ à la surface libre en dérivant $(3.22)$ et en introduisant le résultat dans (3.24).

Après réarrangement, on obtient la répartition de la composante normale de la vitesse relative du fluide (et donc le flux) à la surface du cartilage, sous la forme

$$
\begin{aligned}
\overrightarrow{\vec{v}}_{n} \equiv \vec{v}-\vec{u}_{t}= & \left.\frac{-k}{2 f}\left(f^{2}-\mathscr{Q} \beta\right) \cos \frac{\pi}{2} \xi\right|_{\zeta=u(t)} \times \\
& {\left[\frac{\operatorname{sh} \frac{\pi}{2}\left(\xi+\xi_{1}\right)}{\left.\sin ^{2} \frac{\pi}{2} \zeta\right|_{\xi=u(t)}+\operatorname{sh}^{2} \frac{\pi}{2}\left(\xi+\xi_{1}\right)}\right.} \\
& \left.-\frac{\operatorname{sh} \frac{\pi}{2}\left(\xi-\xi_{2}\right)}{\sin ^{2} \frac{\pi}{2} \zeta \mid \quad \operatorname{sh}^{2}\left(\xi-\xi_{2}\right)}\right]
\end{aligned}
$$

ce qui sera utilisé comme condition aux limites du flux transversal de fluide entrant dans l'espace intraarticulaire. Cette analyse fait l'objet du paragraphe suivant.

Transport des molécules de mucine dans l'espace intra-articulaire

Maroudas (1967) a estimé que l'espace intraarticulaire, lors de sa "fermeture" était de l'ordre de 5000 à $10000 \AA$. Le transport forcé des mucopolysaccharides entre les surfaces articulaires opposées peut être analogue, au niveau de l'ultrastructure, au transport de solutés dans l'eau épithéliale par l'intermédiaire de canaux longs et étroits, ouverts à une extrêmité $\xi=\xi_{2} \equiv \bar{\xi}$ et fermés de l'autre $\xi=0$, cette dernière étant associée à une ligne de symétrie à travers la zone chargée, à l'endroit où la direction de la composante tangentielle de la vitesse du fluide change de signe (Fig. 4).

Le transport actif du soluté dans de telles structures plissées mène à la formation de gradients osmotiques stationnaires, qui dépendent de cinq paramètres :

1) la longueur du canal (dans la zone chargée)

2) la hauteur du canal (qui varie dans le temps lorsque les surfaces articulaires s'approchent et s'éloignent)

3) la perméabilité du fluide à travers les parois du canal cartilagineux

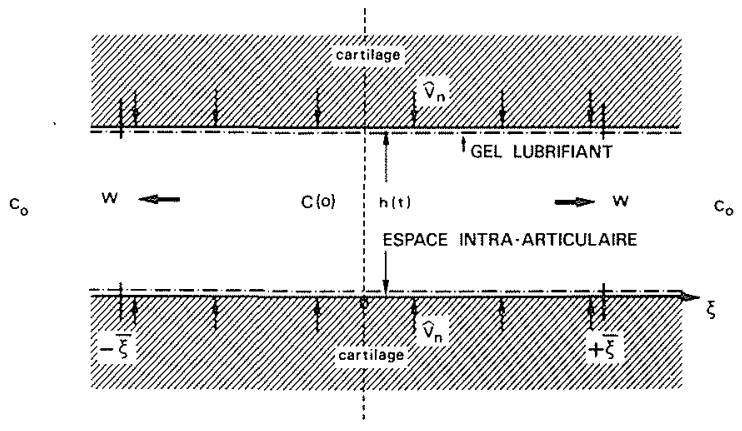

Figure 4 - Transport des macromolécules dans l'espace intraarticulaire.

4) le coefficient de diffusion

5) la vitesse de convection du soluté

Le transport d'eau est à la fois actif, à cause du rapprochement forcé des surfaces cartilagineuses durant l'action de la charge, et passif comme conséquence secondaire des gradients osmotiques locaux établis par le transport actif du soluté de mucopolysaccharides.

On étudie dans ce paragraphe le couplage eau-soluté, en vue d'une détermination du taux de "gélification" des macromolécules pour former une couche lubrificatrice. Selon les théories de la lubrification limite, cette "couche" s'ancrerait dans les fibres de collagène de la surface articulaire, fournissant ainsi le facteur dit de "lubrification".

Un problème analogue a été abordé par Diamond et al. (1967) pour décrire le transport dans les canaux épithéliaux, sous une forme mathématique simplifiée, que l'on généralise ici afin de tenir compte des variations temporelles de la hauteur du canal. La concentration $C$ du soluté sortant de l'extrémité "ouverte" - correspondant au bord de la zone articulaire soumise à la charge peut être inférieure ou supérieure à $C_{0}$, l'osmolarité de la mucine du fluide synovial au-delà de la zone de contact articulaire. La valeur de $C$ sera fonction des cinq paramètres cités ci-dessus.

Le cartilage normal est perméable à l'eau et aux solutés de faible poids moléculaire $M$ jusqu'à une valeur de $M \sim 70000$, excluant ainsi le passage d'hyaluronate $\left(M \sim 2 \times 10^{6}\right)$, qui est par conséquent retenu dans l'espace articulaire. Cette accumulation d'hyaluronate constitue le "gel" qui s'établit à une concentration critique d'environ $1 \%$ (Maroudas 1969), ce qui fournit le facteur lubrifiant de surface (Radin et Paul 1972) essentiel au fonctionnement normal de l'articulation.

On considère un canal bi-dimensional quasi-uniforme de hauteur adimensionnelle $h(t)=h^{\prime}(t) / h_{0}^{\prime}$ et de longueur $\bar{\xi}(t)$. La conservation de masse du fluide dans le canal (cf. Fig. 4) s'exprime par:

$$
\frac{d h}{d t}+\frac{d}{d \xi} h w=-2 \hat{\vec{v}}_{n}
$$

où $w$ est la vitesse axiale du fluide dans le canal, moyennée sur la section $h$, et $\hat{v}_{n}$ la vitesse du fluide entrant transversalement à travers la paroi du canal, suivant l'analyse exposée au paragraphe précédent. Le taux de variation de la hauteur du canal $d h / d t$ est égal 
à $V_{0}$, vitesse de rapprochement des surfaces articulaires lors des phases de charge.

Les variations de concentration du soluté de mucine dans le canal proviennent de l'équilibre quasi-unidimensionnel entre les transports par convection et diffusion

$$
\frac{d C}{d t}+w \frac{d C}{d \xi}=D \frac{d^{2} C}{d \xi^{2}}
$$

où $D$ désigne le coefficient de diffusion des molécules de mucine dans le fluide synovial.

A l'axe de symétrie $\xi=0,\left(\left|\xi_{1}\right|=\left|\xi_{2}\right|=\bar{\xi}\right.$ pour cet exemple)

$$
w=0, \quad C=C(0)
$$

tandis qu'à l'extérieur de l'espace chargé, $\xi=\bar{\xi}, C \rightarrow C_{0}$.

Initialement, $C=C_{0}$ dans l'ensemble du canal lorsque $t=0$. Pour des valeurs faibles du nombre de Peclet $P=V_{0} h / D \sim 0,25$ on peut démontrer que la convection est largement dominée par la diffusion, cette dernière étant suffisamment rapide pour rendre uniforme la concentration, et pour minimiser les effets non-stationnaires.

Par conséquent, la forme stationnaire de l'équation (3.27), liée aux conditions (3.28) aux limites, permet de calculer la concentration $C$ à l'aide de deux quadratures successives

$$
C(\xi, t)=C(0)+\frac{d C(0)}{d \xi} \int_{0}^{\xi} e^{\frac{1}{D} \int_{0}^{\xi^{p}} w d \xi^{\prime}} d \xi
$$

en fonction de la vitesse axiale $w$ dans le canal, obtenue par une simple intégration de l'équation (3.26)

$$
w=\frac{2}{h} \int \hat{v}_{n} d \xi-V_{0} \xi / h+C_{1}
$$

où $C_{1}$ est évalué à partir de la première condition (3.28), et le terme intégré à partir de (3.25), constituant la solution de l'analyse du transport au sein du cartilage.

Après un réarrangement, on obtient, sous forme analytique, une expression décrivant la vitesse du fluide dans le canal

$$
\begin{aligned}
w(\xi ; t) & =\frac{+k\left(F^{2}-\mathcal{D}\right)}{\pi h F(t)}\left(\log \frac{\operatorname{ch} \frac{\pi}{2}(\xi+\bar{\xi})-\cos \frac{\pi}{2} \xi}{\operatorname{ch} \frac{\pi}{2}(\xi+\bar{\xi})+\cos \frac{\pi}{2} \zeta}\right. \\
& \left.-\log \frac{\operatorname{ch} \frac{\pi}{2}(\xi-\bar{\xi})-\cos \frac{\pi}{2} \xi}{\operatorname{ch} \frac{\pi}{2}(\xi-\bar{\xi})+\cos \frac{\pi}{2} \zeta}\right)+V_{0} \xi / h(3.31)
\end{aligned}
$$

qui vérifie la condition de symétrie $w(0, t)=0$. Le premier terme du nombre droit de (3.31) correspond au flux transversal dans le canal dû à l'exudation du fluide aqueux des pores du cartilage chargé, alors que le second terme est associé avec l'approchement des deux surfaces articulaires. On peut démontrer que ce dernier terme domine sur $99 \%$ de la longueur du canal.

Pour une valeur typique de $\bar{\xi}$ de 6,25 , la variation de la vitesse à la paroi nominale $\xi=0$ peut s'écrire sous la forme approximative simplifiée

$$
w \simeq \frac{k\left(F^{2}-\mathscr{S} \beta\right)}{\pi h F(t)} \log \frac{\operatorname{ch} \frac{\pi}{2}(\bar{\xi}-\xi)+1}{\operatorname{ch} \frac{\pi}{2}(\bar{\xi}-\xi)-1}+\frac{V_{0} \xi}{h}
$$

On désigne une concentration initiale $C_{0}$ correspondant à une hauteur $h_{0}$ du canal avant le rapprochement des surfaces articulaires, ainsi qu'une concentration $C(0)$ à $\zeta=0$ (cf. Fig. 4).

Par la condition de la conservation de masse des macromolécules, on obtient

$$
\begin{gathered}
C_{0} h_{0}=C(0) \cdot h(t) \\
\frac{C(0)}{C_{0}}=\frac{1}{1-\frac{V_{0}}{h_{0}} t}
\end{gathered}
$$

d'où

représente la variation temporelle de la concentration $C$ au centre de la zone de contact, en fonction de la vitesse $V_{0}$ de rapprochement des deux surfaces (Fig. 5).

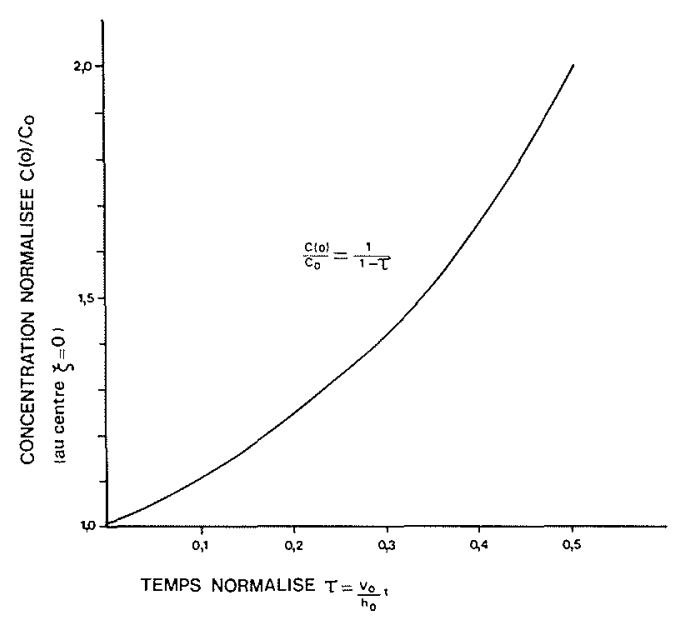

Figure 5 - Accumulation de polysaccharides au centre de la zone de contact.

Une solution approchée de (3.29) qui satisfait aux conditions limites (3.28) et (3.34) est obtenue sous la forme

$$
\frac{C}{C_{0}}=\frac{1}{1-\tau}\left[1-\tau e^{-a(\bar{\xi}-\xi)}\right]
$$

en fonction $\mathrm{du}$ temps adimensionnel $\tau=V_{0} t / h_{0}$

Prenant une valeur normale moyenne de $C_{0}=3,7$ $\mathrm{mg} / \mathrm{ml}$, un coefficient de diffusion $D^{\prime} \sim 0,8 \times 10^{-11}$ $\mathrm{m}^{2} / \mathrm{sec}$ (Balazs et al. 1967) et une contrainte moyenne $F$ de l'ordre de $2 \mathrm{MN} / \mathrm{m}^{2}$, selon les mesures récentes de Seedhom et Tsubuku (1977) pour les surfaces patello-femorales, ainsi qu'une vitesse de rapprochement $V_{0}^{\prime} \simeq 20 \mu / \mathrm{sec}$ à une hauteur d'espace intra-articulaire de $5000 \AA$, on peut calculer l'intervalle de temps, à l'intérieur d'un cycle normal de charge et décharge, pendant lequel la concentration $C^{\prime}$ de mucopolysaccharides dépasse sa valeur critique de "gélification". 


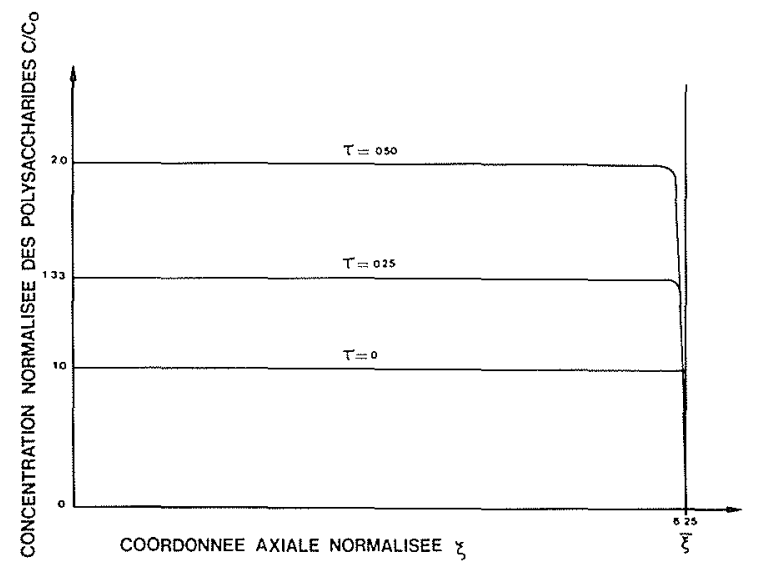

Figure 6 - Répartition calculée de la concentration dans l'espace in tra-articulaire en fonction du temps.

A cause de la valeur relativement faible du coefficient de diffusion $D^{\prime}$, on estime a $\simeq 10^{8}$ dans le terme exponentiel de (3.35). Les résultats (Fig. 6) pour la réparti- tion de concentration des polysaccharides dans le canal intra-articulaire se caractérisent par une quasi-uniformité spatiale, à l'exception des bords de la zone de contact. A $\tau=\tau_{0}, C>C_{\text {crit }}$ marque la première formation d'un tel lubrifiant. Cette valeur critique sera fonction du niveau $p-H$ et peut-être d'autres facteurs biochimiques associés à l'acide hyaluronic, tels que sa dépolymérisation par certains enzymes (cathepsine D ou tripsine)

On peut également associer les premières manifestations de l'ostéoarthrose à une fibrillation du collagène des surfaces articulaires, provoquant ainsi une perte des protéoglycans contenant l'eau qui supporte effectivement la charge.

Le niveau de la concentration ambiante de polysaccharides dans le fluide synovial constitue un troisième facteur qui peut limiter l'achèvement de la valeur critique essentielle pour la formation d'un gel lubrifiant.

Des observations cliniques tendent à confirmer l'irréversibilité de la dégénération ostéoarthritique au-delà d'un certain état. L'évolution de la maladie semble, d'après cette étude, être la suivante :

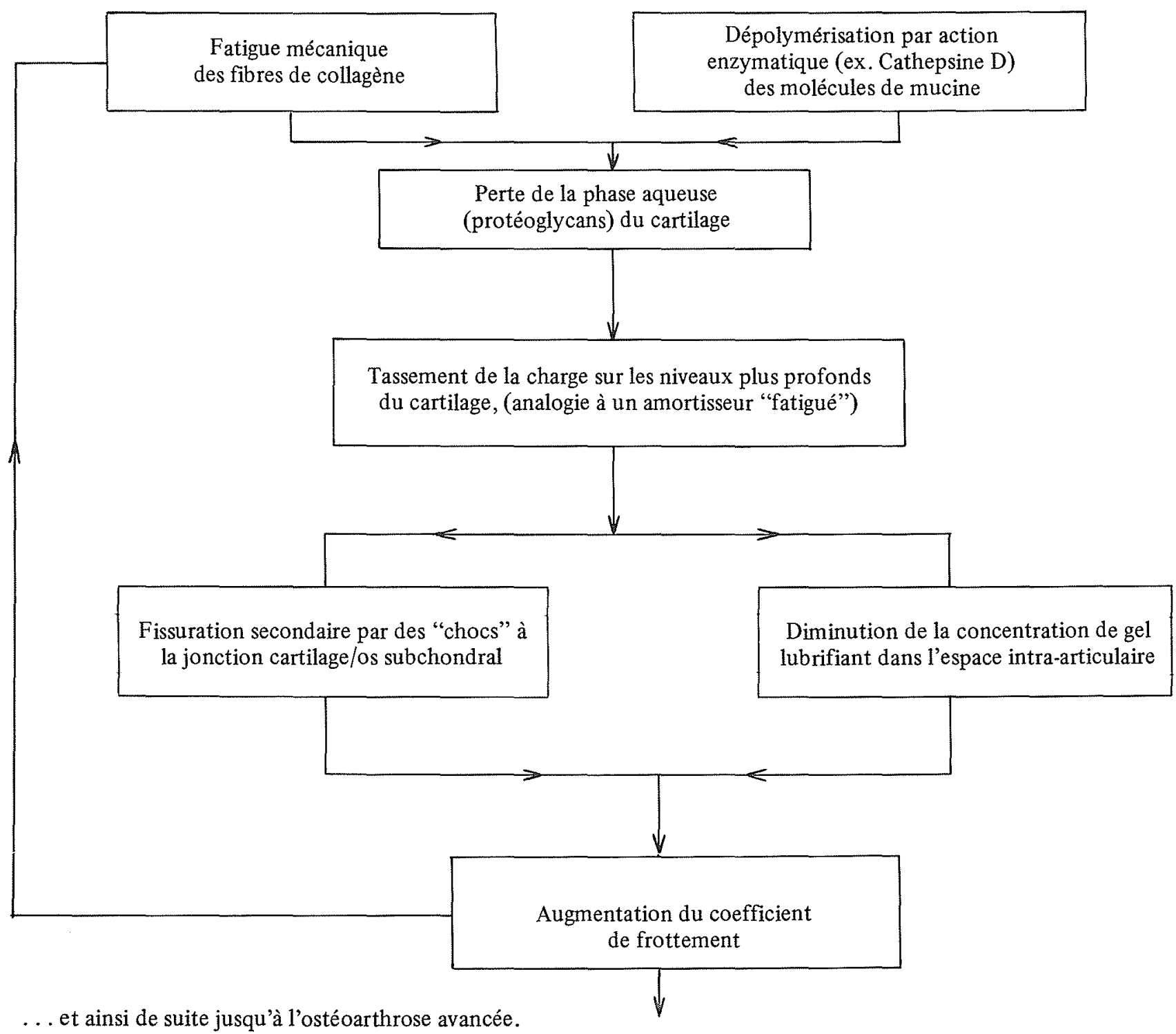


Les prothèses articulaires actuellement utilisées en France ne semblent pas fonctionner selon les principes de la lubrification limite naturelle. Il est possible que leur durabilité restreinte puisse être améliorée par l'application d'une technologie basée sur les mécanismes qui se sont dégagés de cette analyse.

L'auteur exprime ses remerciements à son collègue physiologiste, F. Goubel, pour son aide lors de la préparation du manuscrit.

\section{Références Bibliographiques}

APLEY A.G. (1972) ed. - Modern Trends in Orthopaedics-6 Butterworth, (London).

BALAZS E.A., WATSON D., DUFF I.F., ROSEMAN S. (1967) "Hyaluronic acid in synovial fluid. I. Molecular parameters of hyaluronic acid in normal and arthritic human fluids, Arth.\& Rheum., 10,357.

BIOT M.A., WILLIS D.G. (1957) - The elastic coefficients of the theory of consolidation, J. Appl. Mech. 24, 594.

BIOT M.A. (1963) - Mechanics of deformation and acoustic propagation in porous media, J. Appl. Physics, 33, 1482.

Mc CUTCHEN C.W. (1962) - The frictional properties of animal joints, Wear 5, 1-17.

DARCY H.P.G. (1856) - Les fontaines publiques de la ville de Dijon, Victor Dalmont, Paris.

DIAMOND J.M., BOSSERT W.H. (1967) - Standing-gradient osmotic flow, J. Gen. Physiol. 50, 8, 2061-2083.

FREEMAN M.A.R. (1973) ed. - Adult Articular Cartilage, Pitman Medical (London).

HORI R.Y., MOCKROS L.F. (1976) - Indentation tests of human articular cartilage,J. Biomech 9, 259-269.
KENEDI R.M. (1973) ed. - Perspectives in Biomedical Engg., Mac Milan Press (London)

MANSOUR J.M., MOW V.C. (1976) - The permeability of articular cartilage under compressive strain at high pressures, Journal of Bone \& Joint Surgery, 58-A, no. 4, 509-516.

MAROUDAS A. (1967) - Hyaluronic acid films, Proc. Insth Mech. Engrs, $181 \mathrm{pt} 3 \mathrm{~J}, 122-124$.

MAROUDAS A. (1969) - Studies on formation of hyaluronic acid films, in Lubrification and Wear in Joints, ed. V. Wright, Sector, London, p. 124.

MAROUDAS A. (1973) - Physico-chemical properties of articular cartilage, in Adult Articular Cartilage, ed. M.A.R. Freeman, Pitman Medical, London.

MOW V.C., MANSOUR J.M. (1977) - The nonlinear interaction between cartilage deformation and interstitial fluid, $J$. Biomech 10, 31-39.

NOWINSKI J.L. (1971) - Bone articulations as systems of poroelastic bodies in contact, AIAA Journal 9, 1, 62-67.

RADIN E.L., PAUL I.L. (1972) - A consolidated concept of joint lubrification, J. Bone \& Joint Surgery, 54-A, no. 3, 607-616.

SEEDHOM B.B., TSUBUKU M. (1977) - A technique for the study of contact between visco-elastic bodies with special reference to the patello-femoral joint, $J$. Biomechanics, 10, 253-260.

TORZILLI P.A., MOW V.C. (1976a) - On the fundamental fluid transport mechanisms through normal and pathological articular cartilage during function. Part I: The formulation, J. Biomech. 9, 541-552.

TORZILLI P.A., MOW V.C. (1976b) - On the fundamental fluid transport mechanisms through normal and pathological articular cartilage during function-II. The analysis, solution and conclusions, J. Biomech. 9, 587-606.

WEIGHTMAN B. (1976) - Tensile fatigue of human articular cartilage. J. Biomech, 9, 193-200.

WRIGHT V. (1969) ed. - Lubrication and Wear in Joints, Sector Publ. (London).

\section{Discussion}

En ouvrant la séance, le Président avait observé que les deux premières communications - celle de M. RENAUDEAUX et celle de M. COLLINS - abordaient des problèmes très comparables, l'une sur le plan expérimental, l'autre sur le plan théorique. Il avait proposé de les écouter l'une après l'autre et d'en regrouper la discussion.

Après avoir remercié M. RENAUDEAUX et M. COLLINS, le Président demande à l'assistance de manifester son appartenance au milieu médical ou au milieu technique. Il constate qu'elle comporte une majorité d'ingénieurs.

Il ouvre ensuite la discussion.

M. le Professeur LEMAIRE s'étonne de ne pas avoir entendu parler de la synoviale dont le rôle lui parât pourtant fondamental puisqu'elle secrète le liquide synovial.

M. RENAUDEAUX répond qu'elle n'intervient pas directement mais que ses altérations ont sans doute pour effet de compromettre ou de faire disparaître la lubrification comme cela se passe dans les maladies rhumatismales.

M. PATIN demande si les deux produits essentiels que sont le collagène et la mucine sont parfaitement définis, chimiquement reproductibles et susceptibles de réalisations et d'applications industrielles.

Il évoque entre autre, à cet égard, l'exemple du nylon qui "au fond n'est pas autre chose qu'un assemblage de fibres artificielles conçues, après de longues études, à partir des fibres naturelles que sont les fibres animales".
M. LIORET fait remarquer que, dans un domaine comparable, la Société Dupont de Nemours a consacré des sommes considérables à la mise au point d'un cuir artificiel sans y réussir pleinement.

M. RENAUDEAUX répond que la formule chimique de la mucine est parfaitement connue et que des études sont en cours pour reproduire du collagène de type cartilagineux. Il indique d'autre part qu'on peut espérer trouver dans un avenir prévisible des produits de remplacement qui soient biocompatibles et qui aient des propriétés mécaniques semblables à celles du cartilage. Il mène une étude dans ce sens en collaboration avec l'Institut de Recherches en Chimie Appliquée (I.R.C.H.A.) et avec I'aide de la D.G.R.S.T. pour trouver des matériaux hydrophiles auxquels on pourrait donner certaines propriétés mécaniques.

M. PATIN demande quelle est la profondeur des vides ou des rugosités au niveau du cartilage.

M. RENAUDEAUX indique que cela est assez mal connu et difficile à mesurer mais que l'on s'accorde généralement sur une profondeur moyenne des rugosités de l'ordre de 1 ou $2 \mu$.

M. AUBERT demande si l'usage du téflon - dont le coefficient de frottement est très faible - a été envisagé.

M. RENAUDEAUX croit savoir que c'est un matériau toxique. Il relate à cette occasion l'expérience des prothèses acieracier et acier-plastique. Les premières, à coefficient de frotte- 
rnent relativement grand, s'usent relativement peu; les secondes, à coefficient de frottement plus faible, s'usent davantage.

Le Président observe d'abord que le téflon n'est pas poreux alors que l'une des originalités du système mécanique articulaire réside dans la présence d'une couche cartilagineuse essentiellement poreuse.

D'autre part il convient, pense-t-il, de bien distinguer deux problèmes: "celui de la prothèse dans lequel on essaie de rem. placer une solution naturelle par une solution dite industrielle et celui que nous essayons de résoudre ici : connaître le mécanisme pour éventuellement l'appliquer, soit dans les prothèses, soit dans l'industrie".

M. BONNIN a bien relevé l'importance de la condition de bio-compatibilité pour la recherche et le choix d'un matériau de substitution. Mais s'agissant d'éventuelles applications inđustrielles, cette condition n'intervient pas et il demande si l'on connaît des matériaux à même de remplir correctement des fonctions présentant des propriétés comparables à celles qu sont attribuées au cartilage.

M. RENAUDEAUX répond que, dans un premier temps, le but de ses travaux est de comprendre le "mécanisme"; il recherche un matériau susceptible de l'éclairer sur le "mécanisme" et que, dans cet esprit, il ne s'est pas personnellement préoccupé de bio-compatibilité.

Le Docteur VENET signale qu'une équipe toulousaine s'est livrée à une étude comparative de la synovie de diverses articulations: alors que les personnes âgées ont fréquemment de l'arthrose de la hanche ou du genou, elles souffrent rarement de la cheville. L'observation de la cheville et des genoux sur une vingtaine de cadavres sur des personnes âgées révélait aucune modification de l'articulation de la cheville alors que le genou était généralement arthrosique.

M. RENAUDEAUX lui indique qu'il n'a pas fait d'étude physiologique de ce genre.

Le Docteur ARLET évoque l'œè̀me du cartilage observé avec le développement de l'arthrose pathologique ou traumatique et décrit sous le nom de chondro-malacie - ou ramollissement du cartilage. Il observe finalement que 1" "eau" intervient de diverses façons: tantôt il y a perte de l'eau d'une partie du cartilage, tantôt au contraire il y a excès d'eau en certains points, en particulier semble-t-il dans les zones très traumatisées. lèmes.

Le Docteur SEDEL pense qu'il faut nettement distinguer deux problèmes: d'une part, celui de la chondro-malacie qui, dans l'état actuel des connaissances, serait plutôt du domaine de la biochimie, d'autre part celui du fonctionnement mécanique de l'articulation abordé par les conférenciers. Ces deux types de problème lui paraissent actuellement difficiles à interconnecter.

Le Président suggère de s'en tenir au domaine des conférenciers et demande si des Ingénieurs, familiers des problèmes de lubrification, n'auraient pas des remarques à formuler concernant les deux communications présentées. Il note en particulier l'originalité de l'hypothèse d'un dépôt de gel à la paroi, formulée par M. COLLINS.

M. PATIN répond, comme suit, à cet appel :

On imagine volontiers que, dans les articulations, il y a un frottement comme entre deux surfaces acier sur cuivre ou acier sur acier. Il semble que la friction se fasse en réalité à l'intérieur d'une couche qu'on ne peut pas appeler grasse, mais que l'on appellerait grasse dans un système industriel. Il est bien évident d'autre part que les coefficients de frottement que l'on obtient là ne sont jamais obtenus dans l'industrie, en dehors des phénomènes de frottement hydrodynamique. Or je suis tout à fait de l'avis de $M$. Collins et de $M$. RENAUDEAUX: aux vitesses qui se produisent dans les articulations, il ne peut pas être question de frottement hydrodynamique. Même quand on lance une balle de base-ball, ce doit être de l'ordre de $1 \mathrm{~cm} / \mathrm{sec}$. : cela n'a aucun rapport avec ce que nous décelons dans les paliers. Il y a donc autre chose.

La seule analogie que je pourrais citer est celle qui a été réalisée par la Société Hydromécanique et Frottement, par M.
GAUBET pour les patins de Marcoule, destinés à permettre la mise en tension des câbles de précontrainte. Pour qui connaît la formule du cabestan, les câbles entourant un cylindre constituent une véritable hérésie si l'on n'a pas un coefficient de frottement extrêmement bas. Or pour réaliser un coefficient de frottement bas, le seul moyen est de faire un coussin de graisse : la friction de ces patins se fait alors non pas métal sur métal, mais à l'intérieur de la graisse et simplement par cisaillement de la graisse.

A ma connaissance, c'est le seul exemple d'analogie industrielle avec nos articulations.

On a bien l'impression que, dans l'articulation, le frottement se fait toujours à l'intérieur de la synovie. La théorie de M. COLLINS montre qu'à un moment donné il y a écrasement $\mathrm{du}$ liquide sans qu'il $\mathrm{y}$ ait jamais friction des surfaces solides ou semi-solides entre elles. Celles-ci peuvent se rapprocher à quelques centaines d'angströms, mais sans qu'il y ait contact.

C'est pourquoi je posais la question de la profondeur des rugosités: des rugosités de l'ordre du micron me paraissent un peu incompatibles avec des écrasement amenant la distance à quelques centaines d'angströms.

M. RENAUDEAUX remarque que les rugosités apparaissent au microscope ou au passage d'un stylet mais qu"elles peuvent être masquées par du gel ou autre chose".

M. QUEMADA se déclare frappé par certaines analogies entre les problèmes relatifs à la lubrification des articulations et ceux de la microcirculation. Dans les deux cas il s'agit d'écoulements dans des conduits ou des "espaces" dont l'ordre de grandeur est comparable à celui des particules étudiées. Un régime du type biphasique s'établirait avec une zone centrale où se concentrent ces particules et une zone périphérique appauvrie.

Le profil des vitesses comporte une partie centrale relativement plate avec une viscosité importante se raccordant à la paroi par un gradient de vitesse plus important. Le bilan de l'opération est que les pertes d'énergie sont finalement réduites.

Ainsi observe-t-on une réduction apparente de viscosité au fur et à mesure que le rayon du vaisseau diminue. Dans un tel schéma, ajoute M. QUEMADA, les rugosités "piègeraient" le film du fluide - - de l'eau en l'occurence -- collant aux parois et le film central, concentré en mucine, se tiendrait à une distance à peu près moyenne entre ces parois.

M. QUEMADA rappelle, ̀̀ ce sujet, que certaines procédures de mesure de viscosité du sang comportent l'utilisation de viscosimètres à parois rainurées "pour être sûr que la formation d'une couche plasmotique sans particules ne viendrait pas fausser la mesure".

M. PATIN rappelle, à propos du rôle des rugosités, que l'utilisation des cavités pour le graissage est bien connue en mécanique et couramment exploitée dans le "grattage" des machines-outils.

Concernant les articulations, il se demande si leur fonctionnement ne comporte pas un effet de pompage: Est-ce que dans la marche par exemple, demande-t-il, il n'y a pas successivement rapprochement des surfaces quand on appuic, et éloignement de ces surfaces sous l'effet du poids de la jambe? Le fait est, précise-t-il que quand on reste debout sur une jambe il semble qu'au bout d'un certain temps il n'y a plus de synovie : ce serait la raison de la fatigue.

M. BONIS se demande de son côté quel est le rôle des longues fibres dans l'écoulement de fluides comme le sang ou la synovie: restent-elles mélangées au sein du fluide? S'imbriquent-elles vers la paroi?

M. RENAUDEAUX intervient finalement pour indiquer qu'à ses yeux il n'est pas possible de comparer l'écoulement du sang et celui de la synovie : les ordres de grandeur ne sont pas du tout les mêmes. Dans la synovie, il n'est pas question de parler de profils de vitesses: on ne connâ̂t ni l'épaisseur du film, ni les vitesses d'écoulement. Par contre, en ce qui concerne les rugosités elles n'interviennent pas dans l'écoulement.

M. le Président conclut qu'il y a là des problèmes à examiner plus à fond. 